\title{
Hadron mass generation and the strong interaction
}

\author{
Hans-Peter Morsch ${ }^{* \dagger}$ \\ Institut for Nuclear Studies, Pl-00681 Warsaw, Poland \\ E-mail: h.p.morsch@gmx.de
}

Based on a Lagrangian with a coupling of two gauge bosons to $J^{\pi}=0^{+}$(the quantum numbers of the vacuum) with subsequent creation of elementary fermion-antifermion $(q \bar{q})$ pairs, a model is presented, in which hadrons are created, which couple directly to the absolute vacuum of fluctuating boson fields. By self-consistency requirements the confinement potential as well as densities and masses of scalar and vector states are generated which are in good agreement with the known meson "flavour families" $\omega, \Phi, J / \Psi$, and $\Upsilon$. The sum of the corresponding fermion-fermion potentials can be related to a vacuum potential sum rule, which supports the conjecture that the above flavour states are the eigenstates of the system. In comparison with potential models additional states are predicted, which can explain the large continuum of scalar mesons in the low mass spectrum and also new states detected recently in the charm region.

To satisfy the self-consistency requirements the boson-exchange interaction has to be cut for $r \rightarrow 0$. Transformed to $Q$-space this yields a coupling $\alpha_{s}(Q) \rightarrow 0$ for $Q \rightarrow \infty$ quite similar to that extracted from QCD. However, compared to asymptotic freedom in QCD (explained by spin effects), this effect may be understood by a matching of the effective interaction to the two-boson density.

Apart from mesons, stable baryons have been generated by interference of $(q \bar{q})^{3}$ and $(q \bar{q})^{5}$ production. For the nucleon this leads to a density which is consistent with the electromagnetic form factor deduced from electron scattering. To achieve self-consistency a scalar component is needed also, which turns out to be in agreement with the scalar nucleon $\sigma$-term. Compared to mesons the deduced 'confinement' potential for the nucleon is more shallow but with a steeper slope at large radii giving rise to a large stability.

Interestingly, the structure of baryon-antibaryon production in the present model shows a possible mechanism for the striking matter-antimatter non-equilibrium in the universe and the BIG BANG explosion.

European Physical Society Europhysics Conference on High Energy Physics, EPS-HEP 2009, July 16 - 222009

Krakow, Poland

\footnotetext{
*Speaker.

${ }^{\dagger}$ Postal address: Institut für Kernphysik, Forschungszentrum Juelich, D-52425 Juelich, Germany
} 
In the hierarchy of quantum systems hadrons represent the smallest complex substructures known inside of atoms and nuclei. This is supported by the property of asymptotic freedom [1] of the strong interaction. Therefore, hadrons can be related directly to the absolute vacuum of fluctuating boson fields (with average energy $\bar{E}_{v a c}=0$ ) if the elementary fermion masses are zero. In the current Standard Model the masses of the elementary fermions are nonzero and are generated by the coupling to scalar Higgs fields.

To investigate hadron mass generation without hypothetical Higgs fields a model is studied based on a Lagrangian, in which two gauge bosons couple to a scalar field, from which elementary fermion-antifermion $(q \bar{q})$ pairs are emitted. The possibility that the coupling of two vector bosons to $J^{\pi}=0^{+}$may be important for the generation of bound states has been mentioned already by Cornwall [2], but in context to the structure of QCD. Empirically it is known that strong scalar fields play an important role in hadron excitations [3] and scattering [4]; this is not well understood in present hadron structure models.

Assuming a scalar coupling of two boson $(g)$ fields of the form $g g \rightarrow(q \bar{q})^{n}$ we write the Lagrangian in the form

$$
\mathscr{L}_{S I}=\bar{\Psi} i \gamma_{\mu} D^{\mu} \Psi-\sum_{n} \frac{1}{\tilde{\mu}^{(3 n-2)}} D_{\mu} D^{\mu}(\bar{\Psi} \Psi)^{n}-\frac{1}{4} F_{\mu v} F^{\mu v},
$$

with $\Psi$ being the elementary fermion wave functions, $D^{\mu}$ the covariant derivative $D^{\mu}=\partial^{\mu}-i g_{s} A^{\mu}$, $F^{\mu v}$ the Abelian field strength tensor $F^{\mu \nu}=\partial^{\mu} A^{v}-\partial^{v} A^{\mu}$, and $A^{\mu}$ the gauge fields; further $\tilde{\mu}$ represents a mass.

The structure of $\mathscr{L}_{S I}$ implies a colour neutral coupling of the two gauge fields without colour. Hence, the symmetry of our model is simply isospin SU(2): two elementary fermions $(q)$ with different charge ( 0 and 1$)$ and one vector boson. Because of the need for massless fermions (as discussed below) also the flavour degree of freedom is missing. By the coupling of two vector bosons to $J^{\pi}=0^{+}$the Lagrangian has no chiral symmetry, leading naturally to a sequence of hadronic states as observed experimentally.

The second term in eq. (1) can contribute only, if there is spacial overlap of two boson fields. Therefore, for the study of the mass problem we can reduce our description to three dimensions and study solutions of $\mathscr{L}_{S I}$ within a time-independent Hamiltonian approach.

Due to overlap of two boson fields (given by an extended 2-boson density $\rho_{\Phi}(r)$ ) the recoiling fermion fields are also smeared out giving rise to a fermion density $\rho_{q}(r)$, which, however, cannot exceed $\rho_{\Phi}(r)$. Assuming a "fundamental" state with $J^{\pi}=0^{+}$this yields a constraint

$$
V_{q q}(r)=N \rho_{\Phi}(r)
$$

where $V_{q q}(r)$ is a scalar q-q potential given by folding the one-boson exchange interaction $V_{1 g}(r)$ (between fermions) over the fermion distribution $\rho_{q}(r)$. Having negative intrinsic parity the emitted fermions have to be coupled in a relative p-state, requiring a p-wave distribution of the fermions $\rho_{q}^{p}(\vec{r})=\rho_{q}(r) Y_{1, m}(\theta, \phi)$. This leads to

$$
V_{q q}(r)=\int d r^{\prime} \rho_{q}^{p}\left(\vec{r}^{\prime}\right) Y_{1, m}\left(\theta^{\prime}, \phi^{\prime}\right) V_{1 g}\left(r-r^{\prime}\right) .
$$

$V_{1 g}(r)$ can act only within the density $\rho_{\Phi}(r)$ and has therefore to be cut for large radii. Further, eq. (2) requires, that it has to go to zero for $r \rightarrow 0$, so we use a form $V_{1 g}(r)=f_{a s}(r)\left(-\alpha_{s} / r\right) e^{-c r}$ 

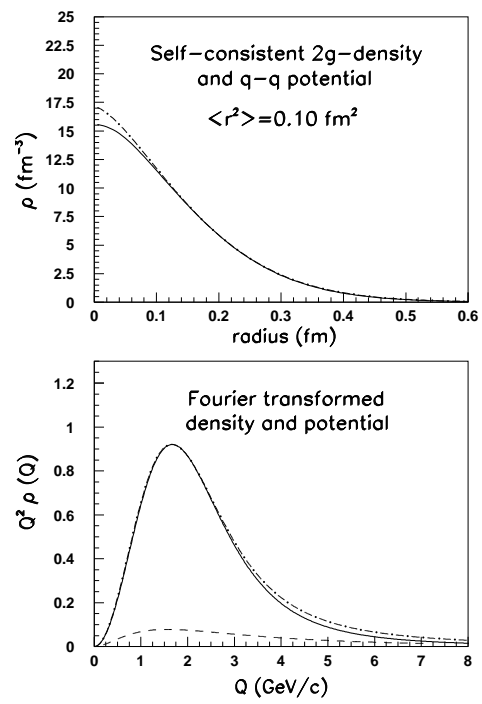

Figure 1: Upper part: Self-consistent solution for a scalar state with two-boson density and q-q potential given by dot-dashed and solid lines, respectively. Lower part: Same as in the upper part but transformed to $Q$-space (multiplied by $Q^{2}$ ). The lower dashed line corresponds to a calculation assuming a mass of $1.4 \mathrm{GeV}$ for the elementary fermions.

with $f_{a s}(r) \rightarrow 0 r \rightarrow 0$. This function as well as the slope parameter $c$ was determined in a selfconsistent fit of the 2-boson density. The p-wave character of $\rho_{q}^{p}(\vec{r})$ gives rise to the constraint $<r_{q}>=\int d \tau r \rho_{q \bar{q}}(r)=0$ and thus $\rho_{q}(r)=\sqrt{3}(1+\beta \cdot d / d r) \rho_{\Phi}(r)$, where $\beta$ is determined from the condition $\left.<r_{q \bar{q}}\right\rangle=0$.

From the different relations between $\rho_{\Phi}(r)$ and $\rho_{q}(r)$ the two-boson density is completely determined. Self-consistent solutions are obtained asuming a form

$$
\rho_{\Phi}(r)=\rho_{o}\left[\exp \left\{-(r / a)^{\kappa}\right\}\right]^{2} \text { with } \kappa \sim 1.5
$$

and an interaction cut-off parameter $c$ which yields a mean square radius of the effective interaction about 60-80\% larger than $\left\langle r_{\Phi}^{2}\right\rangle$. The consistency of $\rho_{\Phi}(r)$ and $V_{q q}(r)$ is shown (in r- and Qspace) in fig. 1. We observe a quantitative agreement for radii larger than $0.1 \mathrm{fm}$. The localisation in space indicates a stationary (mesonic) system, but the emerging fermion pair can couple again to two bosons giving also rise to stable two boson systems.

In Q-space the process $g g \rightarrow q \bar{q}$ is elastic and consequently the created $q \bar{q}$-pair has no mass, yielding also in this frame a consistent description of density and potential, see lower part of fig. 2 . However, if we take a finite mass of the elementary fermions of $1.4 \mathrm{GeV}$ (such a mass has been assumed in potential models [5] for systems of similar size), the dashed line in the lower part of fig. 2 is obtained and no self-consistent solution is possible. Thus, our solutions require massless fermions and consequently the deduced hadronic systems can be related directly to the absolute vacuum of fluctuating boson fields.

For a density $\rho_{\Phi}(r)$ with finite mean square radius as shown in fig. 1 a self-induced binding potential $V_{\Phi}(r)$ can be obtained by solving a Schrödinger equation. This is given in the upper part 


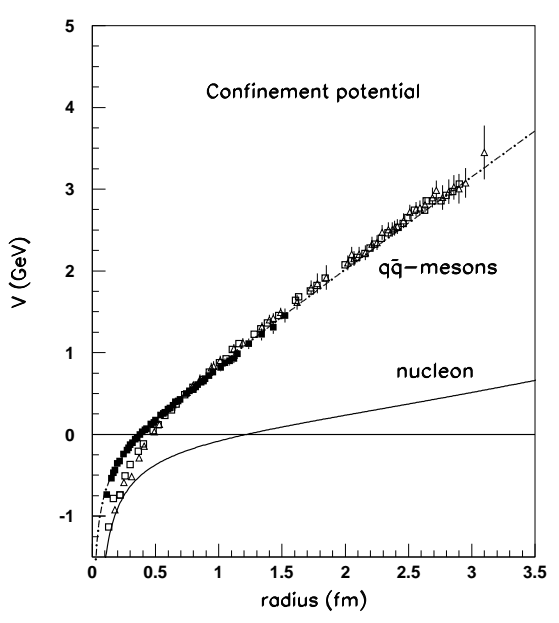

Figure 2: Confinement potential from lattice calculations [6] in comparison with our results. The corresponding potential for the nucleon is shown also.

of fig. 2, which has the same form as the known confinement potential $V_{\text {conf }}=-\alpha_{s} / r+b r$ deduced from potential models [5] and the lattice data of Bali et al. [6]. Further, this potential is quite similar for other solutions of smaller or larger radius and can therefore be identified with the 'universal' confinement potential.

To determine bound state energies of basic scalar and vector $q \bar{q}$-states, binding in the selfinduced confinement potential but also in the q-q potential has to be considered, the latter depending strongly on the radius of the density. For smaller radii a deepening of this potential is observed, which leads to more strongly bound states. The mass of the system is defined by the energy to balance binding. This gives $M_{i}=-E_{q q}+E_{i}$, where $E_{q q}$ and $E_{i}$ are the binding energies in $V_{q q}(r)$ and the confinement potential $V_{\Phi}(r)$.

Discretisation of $q \bar{q}$ states is provided by a vacuum potential sum rule $V_{v a c}(r)=\sum_{n} V_{q q}^{n}(r)$ giving rise to four self-consistent solutions below $50 \mathrm{GeV}$. Corresponding vector states are in good agreement with the masses of the strong $1^{--}$mesons (together with their radial excitations) of the "flavour families" $\omega, \Phi, J / \Psi$ and $\Upsilon$.

Importantly, the Fourier transform to $Q$-space of the cut-off function $f_{a s}(r)$ in the effective interaction $V_{1 g}(r)$ yields $V_{1 g}(Q) \rightarrow 0$ for $Q \rightarrow \infty$. This is similar to asymtotic freedom established for QCD [1].

Interference of $(q \bar{q})^{3}$ and $(q \bar{q})^{5}$ creation can lead to stable baryon-antibaryon systems. Monte Carlo simulations for the nucleon (in which the q-momenta are determined randomly) are given in the upper part of of fig. 3, assuming $(q \bar{q}) q$ and $(q \bar{q})^{2} q$ contributions $\left((q \bar{q}) q\right.$ given by solid, $(q \bar{q})^{2} q$ by dashed histogram). The sum is given by the lower solid histogram, which is consistent with a nucleon density (solid line) yielding an electric form factor of the proton (lower part) in agreement with experiment [7]. In this self-consistent fit a small scalar component is added (dot-dashed histogram) which is consistent with the nucleon- $\sigma$ term (not seen in electron scattering). Interestingly, the splitting up of a $q \bar{q}$-pair (of vector structure) under high pressure provides a mechanism, which could be responsible for the transition of antibaryons to baryons in the early 

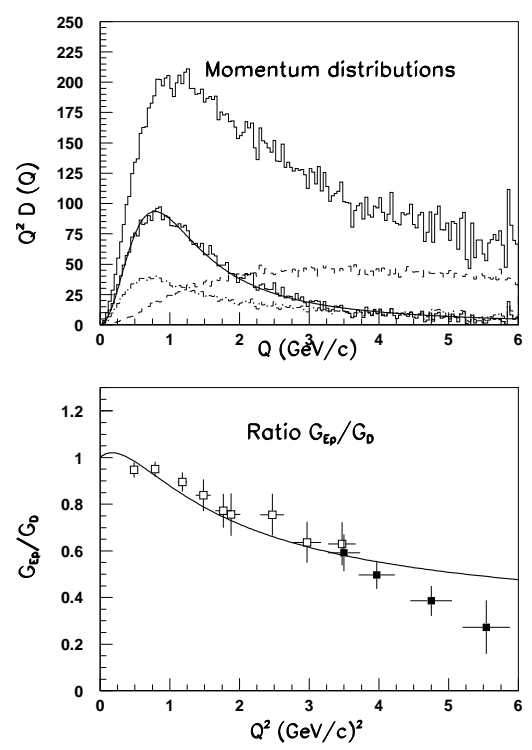

Figure 3: Results of Monte Carlo simulations for the nucleon. The solid line, which is in agreement with the simulation, yields a good description of the electric form factor of the proton (lower part).

universe and the BIG BANG explosion.

For $q \bar{q}$ production more details are given in arXiv:0906.1742[hep-ph].

Many thanks to P. Zupranski for the Monte Carlo code and numerous discussions and B. Loiseau for his help to formulate the Lagrangian. Further, critical comments to my presentation at HEP 2009 from M. Bochicchio are appreciated.

\section{References}

[1] D.J. Gross and F. Wilczek, Phys. Rev. Lett. 30, 1343 (1973); H.D. Politzer, Phys. Rev. Lett. 30,1346 (1973); see also the comments in D.J. Gross, Nobel lecture (2004)

[2] Cornwall, Phys. Rev. D 26, 1453 (1982)

[3] H.P. Morsch and P. Zupranski, Phys. Rev. C 71, 065203 (2005) and refs. therein, H.P. Morsch, Z. Phys. A 350, 61 (1994)

[4] R. Machleidt, Adv. Nucl. Phys. 19 (1989) 189; and refs. therein

[5] R. Barbieri, R. Kögerler, Z. Kunszt, and R. Gatto, Nucl. Phys. B 105, 125 (1976); E. Eichten, K.Gottfried, T. Kinoshita, K.D. Lane, and T.M. Yan, Phys. Rev. D 17, 3090 (1978); S. Godfrey and N. Isgur, Phys. Rev. D 32, 189 (1985); D. Ebert, R.N. Faustov, and V.O. Galkin, Phys. Rev. D 67, 014027 (2003); and refs. therein

[6] G.S. Bali, K. Schilling, and A. Wachter, Phys. Rev. D 56, 2566 (1997)

[7] M.K. Jones, et al., Phys. Rev. Lett. 84, 1398 (2000); O. Gayou, et al., Phys. Rev. Lett. 88, 092301 (2002) 